\title{
A nucleic acid structural alphabet and conformational analyses at dnatco.datmos.org
}

\section{Bohdan Schneider}

\author{
Institute of Biotechnology of the Czech Academy of Sciences, Vestec, Czech Republic \\ bohdan.schneider@gmail.com
}

The experimental models archived in the Protein Data Bank provide a rich source of structural information on proteins and nucleic acids. Complex architectures of RNA molecules as well as non-canonical DNA structures prove that the sugar-phosphate backbone is not a scaffold-like structure more or less passively accommodating to and enabling base pairing and stacking motifs formed by the four nitrogenous bases. In the past, RNA structures attracted more attention [1-5] as their 3D folds are formed by visibly rich ensemble of the backbone geometries. The self-recognition of DNA duplexes posed seemingly fewer challenges to analysis of their structural details. However, a detailed look showed structurally well defined conformers $[6,7]$ that proved useful in discriminating different modes of binding of DNA to transcription factors and the nucleosome core particle in histones [8]. The analysis has shown that differences in the local DNA structure relate to specificity of the binding. In the year 2020, the conformational spaces of DNA and RNA, which were traditionally analyzed separately, were described by one unified set of dinucleotide conformers, which are called $\mathrm{NtC}$, and by a related structural alphabet CANA, Conformational Alphabet of Nucleic Acids [9]. I will briefly describe the principle of fully automated and robust assignment of the $\mathrm{NtC}$ classes and CANA symbols and overview related tools that help to annotate, validate, refine experimental structures, and build computer models of NA molecules. All these tools are feely available at the web service dnatco.datmos.org [10].

[1] Duarte et al. NAR 31:4755 (2003).

[2] Murray et al. PNAS USA 100:13904 (2003).

[3] Hershkovitz et al. NAR 31:6249 (2003).

[4] Schneider et al. NAR 32:1666 (2004).

[5] Richardson et al. RNA 14:465 (2008).

[6] Svozil et al. NAR 36:3690 (2008).

[7] Schneider et al. Acta Cryst D74:52 (2018).

[8] Schneider et al. Genes 8:278 (2017).

[9] Černý et al. NAR 48:6367 (2020).

[10] Černý et al. Acta Cryst D76:805 (2020).

Keywords: nucleic acid structure, DNA structure, RNA structure, dinucleotide conformational class, CANA 